\title{
Patent Litigation and the Power of Correlative Microscopy
}

\author{
Steven L. Goodman ${ }^{1}$, and Jeremy J. Edwards ${ }^{2}$ \\ ${ }^{1}$ Microscopy Innovations LLC, 213 Air Park Rd. Marshfield, WI, 54449, USA. \\ Work completed as 10H Technology Inc., 207 N Allen St. Madison, WI, 53726, USA. \\ 2. Knobbe, Martens, Olson \& Bear LLP, 1717 Pennsylvania Avenue. Washington, DC, 20006, USA.
}

This study shows how correlative microscopy told a compelling story in the context of pharmaceutical patent litigation. The asserted patent related to the use of a "gelling agent" to obtain sustained release of an active ingredient from a tablet. Such tablets form a viscous gel when in contact with water (or gastric fluid). Defendant knew that its tablets did not contain a gelling agent. But in litigation, it is not what you know-it is what you can prove. Moreover, you want to tell a cohesive story, which means anticipating and mitigating any weaknesses in your story.

In microscopy, this is accomplished with correlative methods, wherein multiple instruments modalities and/or sample preparation methods are used. This reduces the potential of improperly interpreting any artifacts of specimen preparation or imaging physics, as multiple methods usually expose any inconsistencies caused by the preparative method or imaging modality. ${ }^{1-2}$

Here, defendant turned to correlative microscopy to characterize the sustained release mechanism of their tablets and demonstrate that their tablets did not contain a gelling agent. It used SEM imaging with various sample preparations, and presented data as $2 \mathrm{D}$ images and as $3 \mathrm{D}$ stereo-pair formats. It also used optical methods to enable imaging of dynamic aqueous interactions, presenting time-lapse video and 3D reconstructions (Table 1). For each of these, defendant compared its tablets to a known gelling tablet.

\begin{tabular}{|l|r|l|l|l|}
\hline Instrumentation & Resolution & Sample Preparation & Observations & Viewing Mode \\
\hline Macroscopic camera $^{1}$ & $0.1 \mathrm{~mm}$ & None & Seconds - Days & Time lapse video \\
\hline Environmental SEM $^{2}$ & $50 \mathrm{~nm}$ & Wet, no coating, 4C, 4T, 20 KeV & Discrete hours & 2D and 3D \\
\hline Field Emission SEM $^{3}$ & $10 \mathrm{~nm}$ & LN2, Freeze-dry, Pt coat, $5 \mathrm{KeV}$ & Discrete hours & 2D and 3D \\
\hline Confocal $^{4}$ & $500 \mathrm{~nm}$ & Dry and in water & Seconds - Minutes & 2D and 3D, Video \\
\hline
\end{tabular}

Table 1: Instruments, approximate resolution, preparations: 1. Dinolite AM413TL-M40. 2. FEI Quanta 200 ESEM. 3. Hitachi S-4100 FE-SEM. 4. BioRad Radiance 2100, Nikon Eclipse TE200, 20x, 0.75 NA.

What defendant generated was compelling. To begin with, the macroscopic imaging clearly showed that defendant's tablet (DT) remained hard and could be cleaved in half after 6 hours of immersion, with no evidence of macroscopic gel formation (Figure 1a). In contrast, the gelling tablet (GT) became a viscous gel (Figure 1b).

But macroscopic imaging could not detect whether DT formed a gel on a microscopic level. To answer this question defendant used ESEM: Samples were immersed in water for various durations, then cleaved for cross-sectional imaging. DT formed $\sim 10-25 \mu \mathrm{m}$ pores at the water interface (Figure 2a), while GT formed a gel that oozed from the tablet edge. Next, defendant examined dry and water-immersed tablets with FE-SEM by freezing in LN2, cryo-cleaving, freeze-drying, and sputter coating. FE-SEM revealed DT to be a compressed aggregate of particles with some voids (Figure 3a). After immersion, DT formed a tortuous tunnel-like porosity extending from the tablet surface towards the core. This porous domain increased from $\sim 150 \mathrm{um}$ deep after $1 \mathrm{hr}$ immersion, to 500 um after 14 
hrs immersion. The core morphology of the GT after 1 hour in water was more uniform, and water interfaces had a structure of radial channels hundreds of microns long. The GT lost its shape after longer immersion. Finally, confocal microscopy dynamically imaged the action of water at the DT surface, and showed pores forming and growing in size and depth within seconds upon water exposure (Figure 4).

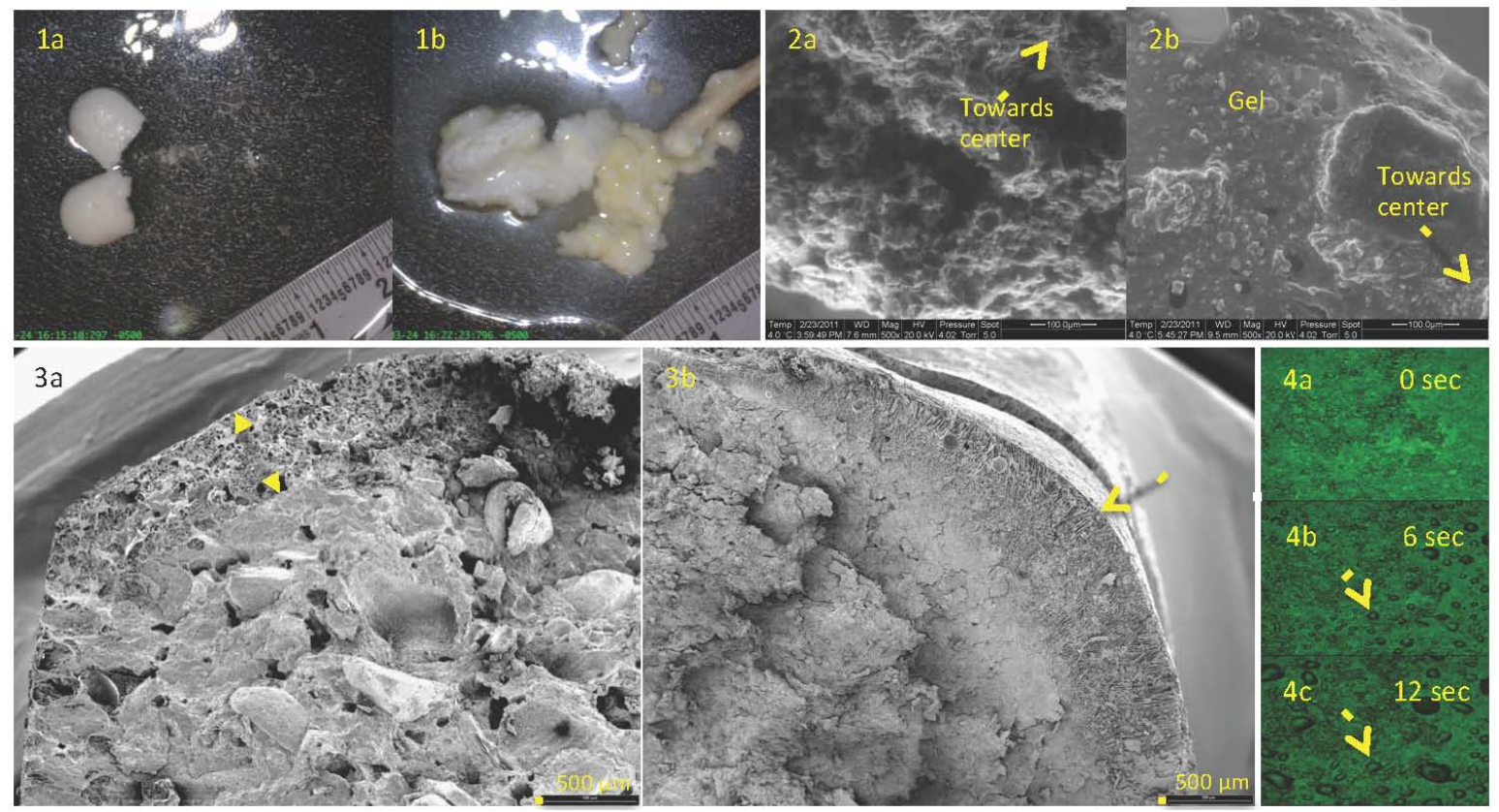

Figure 1: DT (a) and GT (b) in water filled dishes after $6 \mathrm{hrs}$ immersion. Figure 2: ESEM cross-sections of DT (a) and GT (b) after 4 hrs immersion. Arrows show direction towards tablet center core. Figure 3: FE-SEM cross-sections of DT after $14 \mathrm{hrs}$ (a) and GT after $1 \mathrm{hr}$ immersion (b). Double-headed arrow (a) shows depth of complex pore structure at DT surface. Arrow (b) shows radial channels at edge of GT. Figure 4: Confocal image of DT surface before, and at 6 and 12 seconds after water immersion. Arrows point to one of many pores that form and grow over 12 seconds. Field width $606 \mu \mathrm{n}$.

These observations showed that the DT do not form any viscous gel-like phase when in contact with water. To the contrary, the evidence showed that DT provided sustained release by creating a tortuous network of small pores that form by dissolving away soluble components interspersed in the aggregate. This mechanism was consistent with ESEM, FE-SEM and confocal LM observations. In contrast, the GT clearly showed gel formation with all observations, except FE-SEM. However, since FE-SEM preparation utilized freeze-drying, and since gel structures are dependent on water, the observed structure was attributable to an artifact of this dehydration step. This was supported by the absence of any radial channels in ESEM images where the GT remained hydrated. In summary, the microscopy evidence provided defendant with a compelling story that their tablets did not form a gel in contact with water and thus could not infringethe patent.

\section{References:}

[1] Goodman SL, Park K and Albrecht RM in "Colloidal Gold: Methods \& Applications", vol. 3, ed. MA Hayat, p. 369.

[2] Müller-Reichert T and Verkade P (eds). Correlative Light and Electron Microscopy, in "Methods in Cell Biology", volume 111 (2012). 